\title{
Evaluación temprana de técnicas de restauración forestal mediante fluorescencia de la clorofila y diagnóstico de vitalidad de brinzales de encina (Quercus ilex sub. ballota)
}

\author{
Early assessment of forest restoration techniques using chlorophyll fluorescence and \\ diagnosis of oak (Quercus ilex sub. ballota) seedlings vitality
}

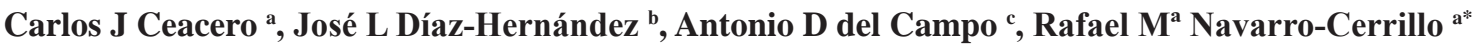

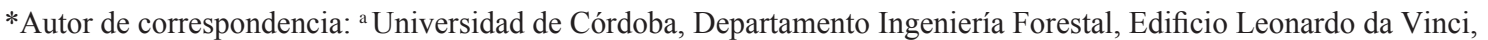 \\ Campus de Rabanales, Carretera Nacional IV, km 396, Aptdo. 3048, 14071 Córdoba, tel.:003495721, ir1nacer@uco.es \\ ${ }^{b}$ IFAPA, Camino del Purchil s/n, Consejería de Agricultura y Pesca, Junta de Andalucía, Aptdo. 2027,18080 Granada. \\ c Universidad Politécnica de Valencia, Departamento Ingeniería Hidráulica y Medio Ambiente, Camí de Vera s/n, 46022 Valencia.
}

\begin{abstract}
SUMMARY
Chlorophyll $a$ fluorescence has been applied to study the evolution of leaf photoprotection processes to dissipate excess absorbed energy that can not be fully utilized in photochemical reactions, and reflects the plants ability to adapt to environmental conditions. Plants environmental conditions can be modified by forest restoration techniques to ensure successful seedlings establishment, hence chlorophyll $a$ fluorescence can be a useful tool for assessing plant response to planting stress. The objective of this paper was to analyze the chlorophyll $a$ fluorescence as an early indicator of oak seedling response to the establishment stress and as a useful tool for the assessment of different competition control and individualized protection techniques (tillage, herbicide, mulch, tree shelter) used in forest restoration. A field experimental plot with these restoration treatments was established and a monthly monitoring of chlorophyll $a$ fluorescence and a vitality analysis were carried out between August 2003 and October 2004 on oak seedlings. Results showed that fluorescence parameters were sensitive to stress conditions prevailing in each analysed period. On the evaluation of herbaceous competition control techniques, no significant results on the chlorophyll $a$ fluorescence were observed. However, tree shelters exercised a protection effect on seedling limiting the photoinhibition processes during spring and winter periods. Finally, protected plants showed major photochemical efficiency than that showed by unprotected ones according to vitality tests. Therefore, the chlorophyll $a$ fluorescence analysis was proved to be a useful tool for early assessment of oak seedlings establishment stress and for the assessment of certain forest restoration techniques, such as those that combine control of herbaceous competition and individualized seedling protection.
\end{abstract}

Key words: photochemical efficiency, photoinhibition, tree shelter, herbaceous competition control.

\section{RESUMEN}

La fluorescencia de la clorofila $a(\mathrm{FC} a)$ se ha aplicado para estudiar procesos de fotoprotección de la hoja para disipar el exceso de energía absorbida que no puede ser totalmente utilizado en las reacciones fotoquímicas, y refleja la capacidad de las plantas para adaptarse a condiciones ambientales. Las técnicas de repoblación forestal modifican las condiciones ambientales para asegurar el éxito en el establecimiento de los brinzales, por lo que la FCa puede ser valiosa para evaluar la respuesta de las plantas al estrés de establecimiento. El objetivo de este trabajo fue analizar la FCa como indicador temprano de la respuesta de brinzales de Quercus ilex sub. ballota al estrés de establecimiento y como herramienta para la evaluación de técnicas de control de la competencia y protección de brinzales (laboreo, herbicida, mulch, tubo invernadero) utilizadas en restauración forestal. Entre agosto de 2003 y octubre de 2004 , se realizó un seguimiento mensual de la FCa y un análisis de vitalidad de brinzales de Q. ilex previamente plantados y sometidos a diferentes tratamientos de restauración en una parcela de ensayo bajo condiciones de campo. Los resultados mostraron que los parámetros de fluorescencia resultaron sensibles a las condiciones de estrés imperantes en cada periodo temporal analizado. En la evaluación de técnicas de control de competencia herbácea, no se observaron resultados significativos en relación con la FCa. Sin embargo, el tubo invernadero ejerció un efecto de protección sobre las plantas que limitó la aparición de procesos de fotoinhibición en primavera e invierno. Las plantas protegidas mostraron mayor rendimiento fotoquímico que las carentes de protección de acuerdo con los análisis de vitalidad. El análisis de FCa mostró ser útil para la evaluación temprana del estrés de establecimiento de Q. ilex y para la evaluación de determinadas técnicas de restauración forestal, como aquellas que combinan el control de la competencia con la protección individualizada de los brinzales.

Palabras clave: rendimiento fotoquímico, fotoinhibición, tubo invernadero, control de competencia herbácea. 


\section{INTRODUCCIÓN}

El objetivo principal de una repoblación forestal es garantizar la supervivencia de los brinzales, especialmente durante la fase crítica del arraigo. En ambientes mediterráneos las plantas deben hacer frente a diferentes tipos de estrés ambiental, entre los que destaca el estrés hídrico. Se ha comprobado la existencia de interacciones entre el déficit hídrico y otros estreses. Así, es un hecho conocido que la respuesta ante el estrés hídrico está estrechamente correlacionada con la respuesta al estrés térmico y lumínico (Valladares y Pearcy 1997, Martínez-Ferri et al. 2000).

La energía solar y en concreto la parte del espectro comprendida entre los 400 y $700 \mathrm{~nm}$ (radiación fotosintéticamente activa) es la radiación utilizada en el proceso de la fotosíntesis para la producción vegetal. Un exceso de luz puede dañar los sistemas fotosintéticos. El fenómeno de fotoinhibición se define como la disminución de la tasa fotosintética o del rendimiento cuántico que se produce como consecuencia de un exceso de radiación (Niyogi 1999). Por tanto, no toda la energía absorbida por la clorofila dentro de las hojas se utiliza durante la fase dependiente de la luz de la fotosíntesis, sino que parte de ella se libera en forma de calor o se re-emite como fluorescencia. $\mathrm{Al}$ ser procesos competitivos, los cambios en las tasas de fotosíntesis tendrán un reflejo sobre la emisión de calor y fluorescencia y lo mismo ocurrirá en sentido contrario. Es por ello, que el estudio de la fluorescencia de la clorofila se considera una herramienta útil para distinguir diferentes efectos (estomáticos y no estomáticos) en la limitación de la fotosíntesis (Maxwell y Jonhson 2000). Diversos investigadores (Baquedano y Castillo 2006, Tambussi 2005) describen diferentes procesos para disipar de forma segura el exceso de energía que se genera en condiciones de estrés (ciclo de las xantofilas, fotorrespiración, reacción de Mehler). Estos procesos evitan fenómenos de fotoinhibición y fotooxidación severos.

A temperaturas fisiológicas, prácticamente toda la emisión de fluorescencia procede de la clorofila a asociada con el fotosistema II. Éste último constituye un importante componente del aparato fotosintético de las plantas, ya que es el lugar donde se producen la foto-oxidación del agua y la reducción de los transportadores electrónicos fotosintéticos, siendo el fotosistema II uno de los primeros procesos del sistema fotosintético afectado por condiciones de estrés, por lo que el análisis de su comportamiento proporciona un diagnóstico temprano del estrés sobre la vegetación. El análisis de fluorescencia de la clorofila $a$ tiene la ventaja de ser una técnica no destructiva, sencilla y de rápida respuesta (Strasser et al. 2000). La mayoría de los estudios de fluorescencia se basan en la respuesta observada cuando la hoja se ilumina rápidamente tras un periodo de adaptación a la oscuridad. La cinética de la respuesta se analiza a través de la denominada curva de Kautsky, que presenta una fase de incremento rápido de la fluorescencia en el primer segundo de iluminación seguida de una fase lenta de declive de la fluorescencia durante varios minutos (Strasser et al. 2000). La fase rápida (designada secuencialmente por las letras O, J, I, P), está relacionada principalmente con eventos primarios del fotosistema II. Strasser y Strasser (1995) formularon un grupo de parámetros de fluorescencia que cuantificaban el flujo de energía a través del fotosistema II a partir del análisis de la cinética de la curva de Kautsky. El parámetro más estudiado es la razón entre la fluorescencia variable a $2 \mathrm{~ms}(\mathrm{Fv})$ y la fluorescencia máxima ( $\mathrm{Fm})$, denominado como $\mathrm{Fv} / \mathrm{Fm}$, que es proporcional a la máxima eficiencia fotoquímica primaria de las hojas (Demming-Adams y Adams 1992). En general, cualquier proceso de estrés como altas y bajas temperaturas, sequía o exceso de luz pueden registrar una disminución de la máxima eficiencia fotoquímica primaria. Sin embargo, el estrés hídrico es el factor más limitante en el descenso de la tasa fotosintética en ambientes mediterráneos. Por esta razón, gran cantidad de trabajos, parte de ellos desarrollados exclusivamente para encina (Quercus ilex sub. ballota [Desf.] Samp.), han centrado su interés en la sensibilidad de este parámetro frente a fenómenos de sequía y estrés hídrico (Ogaya y Peñuelas 2003, Oliveira y Peñuelas 2004, Baquedano y Castillo 2006). Algunos autores proponen un análisis más completo de los parámetros de fluorescencia en lugar del empleo exclusivo de la máxima eficiencia fotoquímica primaria (Force et al. 2003, Christen et al. 2007), como el uso del índice de potencial fotosintético (PIabs) (Strasser et al. 2000). Se trata de un parámetro integrado que incluye tres parámetros independientes: la densidad de centros de reacción totalmente activos, la probabilidad de que un fotón absorbido sea atrapado por el centro de reacción, y la eficiencia de que la energía de excitación mueva un electrón más allá de la quinona $\mathrm{A}$ en la cadena de transporte electrónico. El índice de potencial fotosintético proporciona una información integrada de tres procesos importantes del sistema fotosintético. En primer lugar, al igual que la máxima eficiencia fotoquímica primaria, informa sobre las reacciones fotoquímicas primarias; en segundo lugar, sobre la eficiencia de conversión de energía en la fase oscura de la fotosíntesis; y, finalmente, sobre la densidad de centros de reacción activos en la clorofila. El índice de potencial fotosintético refleja, por tanto, la funcionalidad del fotosistema I y II y proporciona información cuantitativa del estado fisiológico de la planta bajo condiciones de estrés (Strasser et al. 2004).

La hipótesis de este trabajo parte de que cualquier proceso de estrés ambiental puede ser detectado de forma temprana mediante el análisis de fluorescencia, y establece que si los tratamientos de control de la competencia y protección de los brinzales aplicados afectan a las condiciones ambientales que generan estrés en las plantas establecidas y a su respuesta ecofisiológica, estos efectos podrán ser evaluados de forma temprana mediante el análisis de fluorescencia de la clorofila $a$. De esta forma, las técnicas de fluorescencia y el análisis de vitalidad de plantas se con- 
vertirían en herramientas válidas para la evaluación temprana de técnicas de restauración.

De acuerdo con esta hipótesis, los objetivos de este trabajo son: 1) verificar que en las condiciones de ensayo la fluorescencia de la clorofila $a$ puede actuar como indicador temprano del estrés de establecimiento en brinzales de encina; y 2) analizar si la fluorescencia de la clorofila $a$ es particularmente útil para la evaluación temprana de diferentes técnicas de restauración forestal (laboreo, herbicida, mulch, tubo invernadero), mediante el análisis de los parámetros de fluorescencia y los efectos que las técnicas estudiadas ejercen sobre las condiciones de estrés al establecimiento de los brinzales.

\section{MÉTODOS}

Características generales de la parcela de experimentación. La parcela se localizó en el término municipal de Córdoba, en la finca Alameda del Obispo (IFAPA-Córdoba) $\left(37^{\circ} 51^{\prime} \mathrm{N}\right.$ y $4^{\circ} 48^{\prime} \mathrm{O}, 92 \mathrm{~m}$ s.n.m), sobre la terraza inferior del río Guadalquivir, en un terreno dedicado anteriormente al cultivo y producción agraria. La parcela tiene una topografía plana, ligeramente inclinada hacia el río (pendiente $<1 \%$ ), colgada unos $5 \mathrm{~m}$ sobre su cauce y $\sin$ mostrar síntomas evidentes de erosión. Los suelos están dominados por fluvisoles cálcicos con abundantes gravas $\mathrm{y}$ de espesor irregular pero que no superaron los $50 \mathrm{~cm}$. El clima de la zona corresponde a un clima mediterráneo, con una precipitación media anual de $536 \mathrm{~mm}$, con veranos secos y calurosos e inviernos templados (temperatura media anual de $\left.17,6^{\circ} \mathrm{C}\right)$.
En cuanto a las condiciones de estrés ambiental durante el periodo de estudio, en la etapa crítica estival se alcanzaron temperaturas máximas por encima de $40{ }^{\circ} \mathrm{C}$ durante más de 25 días y se acumularon más de 150 días sin $11 u-$ vias significativas entre los meses de abril y septiembre de 2003 (16,8 mm). Durante los meses de invierno, las temperaturas medias ambientales mostraron un amplio rango de oscilación (entre 3 y $12{ }^{\circ} \mathrm{C}$ ) con temperaturas mínimas por debajo de $2{ }^{\circ} \mathrm{C}$ en más de 30 días (figura 1 ).

En enero de 2003 se realizó una plantación manual de encina con planta de una temporada cultivada en contenedores Forespot ${ }^{\circledR}$ de $400 \mathrm{~cm}^{3}$ (altura $11,59 \pm 0,75 \mathrm{~cm}, \mathrm{~N}=80$ y diámetro $4,12 \pm 0,31 \mathrm{~mm}, \mathrm{~N}=80$ ). Previamente a la plantación, la parcela se preparó hasta una profundidad de $50 \mathrm{~cm}$ mediante subsolador de un sólo rejón, y posteriormente se gradeó. Se utilizó un marco regular de plantación de 2 x 1,5 m.

El diseño experimental elegido fue un multifactorial de bloques completos al azar con cuatro repeticiones y 20 unidades muestrales (plantas) por combinación factorial, lo que hace un total de 80 plantas por tratamiento. Se ensayaron tres tratamientos de control de la competencia (laboreo, mulch y herbicida) y sus respectivas combinaciones con la presencia o no de tubo protector, finalmente se introdujo un tratamiento control. Las características de cada uno de los tratamientos ensayados fueron las siguientes:

- Control (C): presupone el mantenimiento de la cobertura herbácea presente en la parcela en el momento de comienzo del experimento.

- Laboreo (L): se llevó a cabo mediante dos pases de cultivador rotatorio sobre tractor de $40 \mathrm{hp}$ hasta una

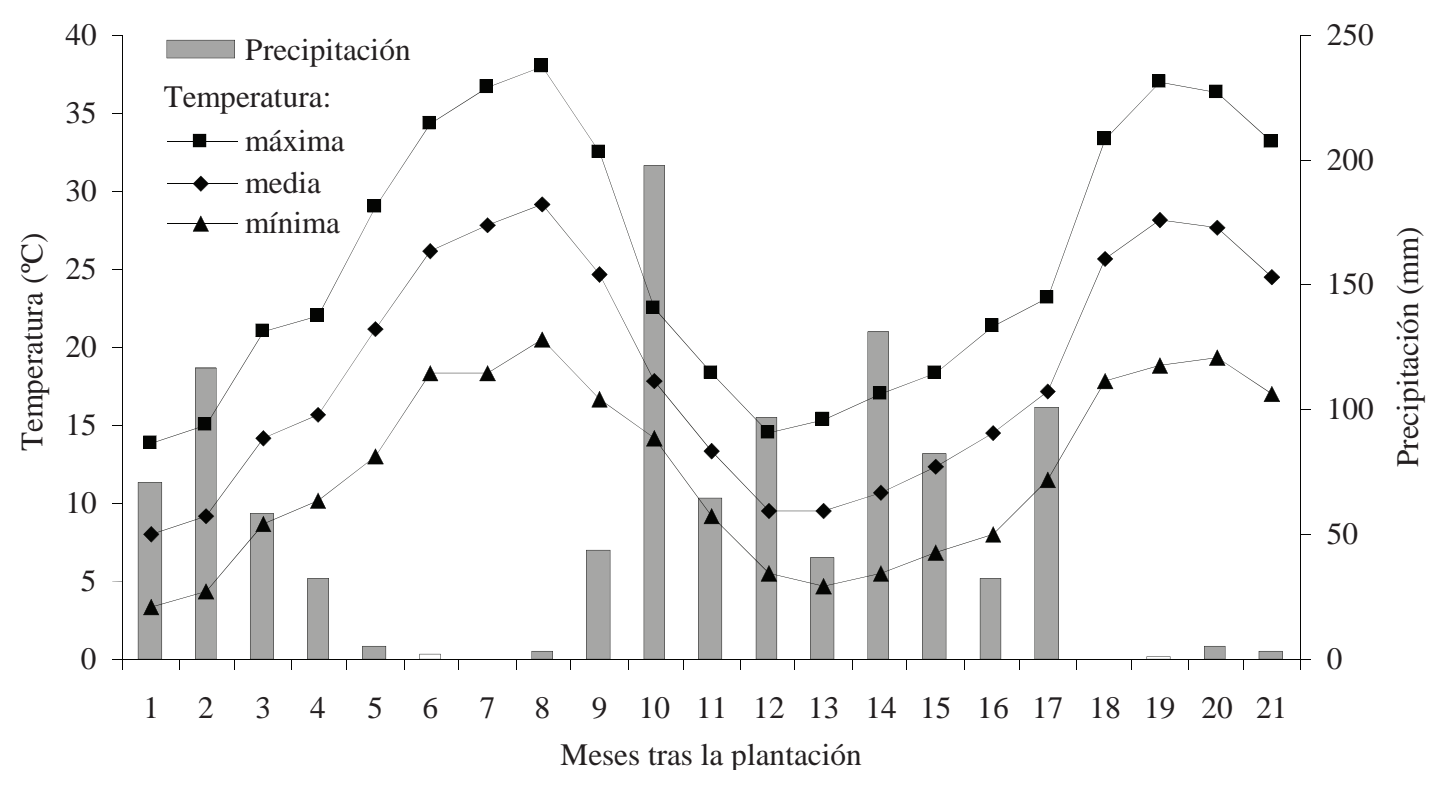

Figura 1. Evolución mensual de variables meteorológicas en la parcela de experimentación durante el periodo de estudio (estación agroclimática de Córdoba, $37^{\circ} 51^{\prime} \mathrm{N}$ y $4^{\circ} 48^{\prime} \mathrm{O}$ ).

Monthly evolution of meteorological variables on the experimental plot during the monitoring period (Córdoba agroclimatic station, $37^{\circ}$ $51^{\prime} \mathrm{N}$ and $\left.4^{\circ} 48^{\prime} \mathrm{W}\right)$. 
profundidad de $5 \mathrm{~cm}$ en el mes de mayo, momento de máxima presencia de herbáceas.

- Cubierta de plástico (M) de $1 \mathrm{~m}^{2}$ de superficie de $40 \mu \mathrm{m}$ de espesor en polietileno negro y enterrado manualmente a $3 \mathrm{~cm}$ de profundidad.

- Herbicida $(\mathrm{H})$ : mediante la aplicación de Oxifluorfen $24 \%$ a una dosis de $2 \mathrm{~kg}$ de materia activa por hectárea. El tratamiento se aplicó con bomba pulverizadora de espalda a principios del mes de mayo.

- Tubo invernadero (T): tubo cilíndrico Fortetub ${ }^{\circledR}$ de $60 \mathrm{~cm}$ de altura y de 86 a $110 \mathrm{~mm}$ de diámetro, fabricado en polipropileno en doble capa inyectado, de color marrón claro y ventilado, con numerosos agujeros dispersos sobre la parte superior del tubo.

Finalmente, durante el mes de marzo de 2004 se repitieron los tratamientos ensayados.

Fluorescencia de la clorofila a. La fluorescencia de la clorofila $a$ se determinó utilizando un fluorímetro Plant Effciency Analyser (PEA, Hansatech Instruments, UK) sobre una muestra de cuatro plantas por tratamiento y con una frecuencia mensual. Las mediciones se realizaron a temperatura ambiente en hojas prendidas al tallo en su tercio superior y sin síntomas visibles de daño. La fluorescencia fue inducida mediante la excitación de un pulso de un segundo de luz roja $(640 \mathrm{~nm})$, suministrado por una luz emitida por seis diodos (LEDs) $\left(600 \mathrm{~W} \mathrm{~m}^{-2}\right.$ o $\left.3.500 \mu \mathrm{mol} \mathrm{m}^{-2} \mathrm{~s}^{-1}\right)$ localizado en un área de $4 \mathrm{~mm}$ de diámetro. El tiempo de adaptación a la oscuridad fue de 30 minutos. La fluorescencia fue detectada utilizando un fotodiodo, después de haber atravesado un filtro (50\% transmitancia a $750 \mathrm{~nm}$ ). Todas las plantas fueron medidas bajo las mismas condiciones.

Se analizaron las curvas de cinética rápida de la fluorescencia de clorofila $a$ mediante la señal de fluorescencia medida al tiempo $50 \mu$ s después de iniciada la iluminación como valor inicial de fluorescencia (Fo). El análisis de fluorescencia de la clorofila $a$ se realizó mediante la aplicación del denominado OJIP test (Strasser et al. 2000), teniendo en consideración la información correspondiente a los diferentes flujos energéticos que se producen en el aparato fotosintético (figura 2, cuadro 1). El programa de análisis empleado para el cálculo de los parámetros OJIP fue Biolyzer (Maldonado-Rodríguez 1999). Del conjunto de parámetros OJIP (cuadro 1), se analizaron la fluorescencia inicial (Fo), la fluorescencia máxima (Fm), la máxima eficiencia cuántica del PSII (Fv/Fm), la eficiencia del transporte de electrones (ETo/ABS) y el índice de potencial fotosintético (PIabs).

Análisis de vitalidad de los brinzales. Se realizó un análisis de vitalidad de la planta (Hermans et al. 2003) para los diferentes tratamientos de control de la competencia contrastados para los dos años de seguimiento del ensayo. Para ello se determinó el valor de PIabs para cada tratamiento [(PIabs)trat] como el valor medio de los brinzales sometidos a un tratamiento concreto a lo largo de los dos

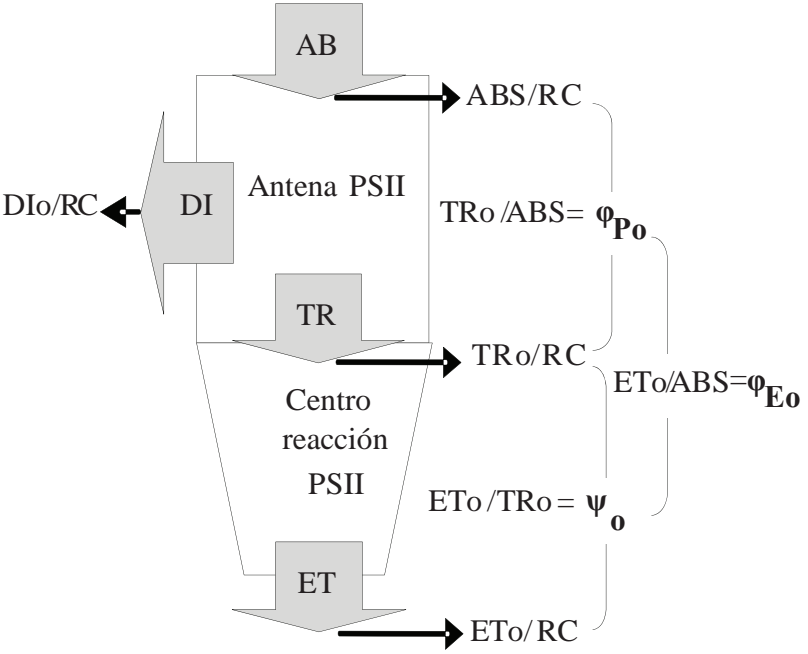

Figura 2. Modelo simplificado de flujos de energía en el aparato fotosintético. ABS se refiere al flujo de fotones absorbido por los pigmentos de la antena ( $\left.\mathrm{Ch}^{*}\right)$ en el fotosistema II. Parte de esta energía de excitación (DI) se disipa como calor y en menor grado como emisión de fluorescencia y la otra parte es canalizada como flujo atrapado TR al centro de reacción y es convertido a energía redox reduciendo al aceptor de electrones $Q_{A}$ a $Q_{A}{ }^{-}$el cual se oxida creando transporte de electrones ET. A partir de estas fluctuaciones de flujo energético se determinan los parámetros de eficiencia cuántica $\left(\varphi_{\mathrm{Po}}, \psi_{\mathrm{o}}, \varphi_{\mathrm{EO}}\right)$ como relación de dos de ellos, tal y como queda definido en el diagrama adjunto. Adaptado de Force et al. (2003).

Simplified models of energy flux of photosynthetic system. ABS: photons flux absorbed by antenna pigments of photosystem II (Ch1*). Part of the excitation energy (DI) is dissipated as heat and fluorescence emission and the rest is directed as retained flux (TR) to the reaction center and transformed into redox energy to reduce electron acceptor QA to QA- and to induce electrons transportation ET. As result of energy flux, parameters of quantic efficiency are determined $(\varphi \mathrm{Po}, \psi \mathrm{o}$, $\varphi$ Eo). Adapted from Force et al. (2003).

años de control y, por otra parte, se calculó un valor de dicho índice para el total de la parcela de ensayo [(PIabs) parc], que se definió como el valor medio de todas las mediciones de PIabs realizadas $(\mathrm{n}=120$ plantas). La relación entre ambos valores (ecuación 1), permitiría clasificar cada tratamiento en tres grupos de calidad, uno por encima del valor medio, otro por debajo y uno coincidente con el valor medio del total de la parcela de experimentación.

$$
\Delta \mathrm{PI}(\mathrm{abs}) \mathrm{re}(\%)=100 \times\left[\begin{array}{l}
\text { (Plabs)trat- } \\
\text { (Plabs) parc }
\end{array}\right] /(\text { Plabs) parc }
$$

Finalmente, se comparó el índice de potencial fotosintético relativo, considerado un indicador de la fuerza motriz fotosintética de una muestra (Driving Force, DFabs $=$ Log PIabs), con el rendimiento relativo del transporte de electrones fotosintético de esa muestra (ETo/ABS)rel (Hermans 
Cuadro 1. Formulación y definición de parámetros OJIP-test.

OJIP-test parameters and equations.

\begin{tabular}{lll}
\hline Parámetros técnicos & & \\
\hline Fluorescencia a $50 \mu \mathrm{s}$ & $\mathrm{Fo}$ & \\
Fluorescencia máxima & $\mathrm{Fm}$ & \\
Fluorescencia variable a $2 \mathrm{~ms}$ & $\mathrm{FV}$ & $=\mathrm{Fm}-\mathrm{Fo}$ \\
Pendiente desde el origen de la fluorescencia & $\mathrm{Mo}$ & $=(\mathrm{F} 300 \mu \mathrm{s}-\mathrm{Fo}) /(\mathrm{Fm}-\mathrm{Fo})$ \\
Fluorescencia variable relativa a $2 \mathrm{~ms}$ & $\mathrm{Vj}$ & $=(\mathrm{F} 2 \mathrm{~ms}-\mathrm{Fo}) /(\mathrm{Fm}-\mathrm{Fo})$
\end{tabular}

et al. 2003). En este caso fue necesaria la determinaron de los valores medios por tratamiento [(PIabs)trat; (ETo/ABS) trat] y para el total de mediciones de la parcela de ensayo en los dos parámetros seleccionados [(PIabs)parc; (ETo/ ABS)parc]. Ambos valores relativos quedaron definidos, respectivamente, de acuerdo a las ecuaciones 2 y 3 .

\section{$\Delta$ (DFabs) rel $=\log ($ Plabs $) r e=$ Log[( Plabs)trat/ (Plabs) parc $]=$ Log(Plabs)trat-Log(PIabs) parc}

$(\mathrm{ETo} / \mathrm{ABS}) \mathrm{re}=\left[\begin{array}{l}(\mathrm{ETo} / \mathrm{ABS}) \text { trat }- \\ (\mathrm{ETo} / \mathrm{ABS}) \text { parc }\end{array}\right] /(\mathrm{ETo} / \mathrm{ABS})$ parc

Tratamiento estadístico. La influencia del factor tratamiento sobre los parámetros de fluorescencia, se analizó mediante un análisis de la varianza (ANDEVA). Con carácter general, cuando estos análisis reflejaron la existencia de diferencias significativas se empleó la prueba de Tukey para la diferenciación en subconjuntos homogéneos de medias. Los datos fueron transformados en aquellos casos en que fue necesario para asegurar el cumplimiento de las exigencias del análisis aplicado: normalidad y homocedasticidad. El nivel de significación utilizado en todos los casos fue $P<0,05$. Todos los análisis estadísticos se realizaron con el paquete estadístico SPSS v.12.0.

\section{RESULTADOS}

Fluorescencia de la clorofila a. La evolución temporal del valor medio de la fluorescencia basal (Fo) reveló diferencias significativas por tratamiento únicamente para el periodo estival del primer año de seguimiento (verano 2003, 
$\mathrm{F}=4,946, P=0,003$ ) (cuadro 2). El tratamiento laboreo con tubo (LT) mostró los valores máximos de la fluorescencia basal $(\mathrm{Fo}=1.268,75)$, contrastando con los obtenidos para los tratamientos individuales $(\mathrm{C}, \mathrm{L}, \mathrm{M}$ y H), que oscilaron entre 500 y 650 (valores relativos). Los mismos resultados fueron obtenidos para la fluorescencia máxima (Fm), si bien en este caso, también fueron significativas las medidas de invierno y primavera del año 2004 (cuadro 2). Durante el verano del 2003, el tratamiento control (C) $(\mathrm{Fm}=799)$ fue significativamente diferente de los tratamientos LT $(\mathrm{Fm}=2.636,75)$ y mulch con tubo $(\mathrm{MT})(\mathrm{Fm}=2.770,75)$, que mostraron los valores máximos. En el periodo invernal fue el laboreo (L) el tratamiento que registró el valor mínimo $(\mathrm{Fm}=1.802)$ mientras los máximos fueron para los tratamientos LT y MT $(\mathrm{Fm}=3.022-3.127)$. En primavera, los tratamientos LT y MT tuvieron valores mayores que los tratamientos individualizados (L, M y H) (cuadro 2).

En relación a los resultados obtenidos para la relación Fv/Fm son destacables los valores correspondientes al verano de 2003, ya que mostraron importantes caídas respecto a los observados en el resto de las medidas para el total de tratamientos ensayados. El valor Fv/Fm mostró diferencias significativas entre los tratamientos para los controles de invierno $(\mathrm{F}=5,165 ; P=0,021)$ y de primavera $(\mathrm{F}=5,524$; $P=0,005)$ del segundo año de seguimiento. En la medida del invierno de 2004, el tratamiento L $(\mathrm{Fv} / \mathrm{Fm}=0,67)$ mostró diferencias significativas respecto a los tratamientos LT $(\mathrm{Fv} / \mathrm{Fm}=0,76)$ y $\mathrm{HT}(\mathrm{Fv} / \mathrm{Fm}=0,75)$. Durante la primavera de ese año, los valores extremos se obtuvieron entre el tratamiento $\mathrm{M}(\mathrm{Fv} / \mathrm{Fm}=0,67)$ y su combinación con tubo $(\mathrm{MT})(\mathrm{Fv} / \mathrm{Fm}=0,77)$ y LT $(\mathrm{Fv} / \mathrm{Fm}=0,77)($ cuadro 2$)$.

Finalmente, el valor del PIabs mostró diferencias significativas entre tratamientos en el control de primavera de 2004 ( $\mathrm{F}=3,987 ; P=0,017)$, siendo los valores máximos para el tratamiento LT $($ PIabs $=21,77)$ y los mínimos para el M (PIabs = 4,22).

Por otro lado, la fluorescencia basal (Fo) mostró diferencias significativas asociadas al factor tubo invernadero para el control estival del año 2003 y los correspondientes al invierno y al otoño del 2004 (figura 3). En todos los casos, los tratamientos combinados con tubo invernadero registraron valores superiores a los tratamientos sin tubos,
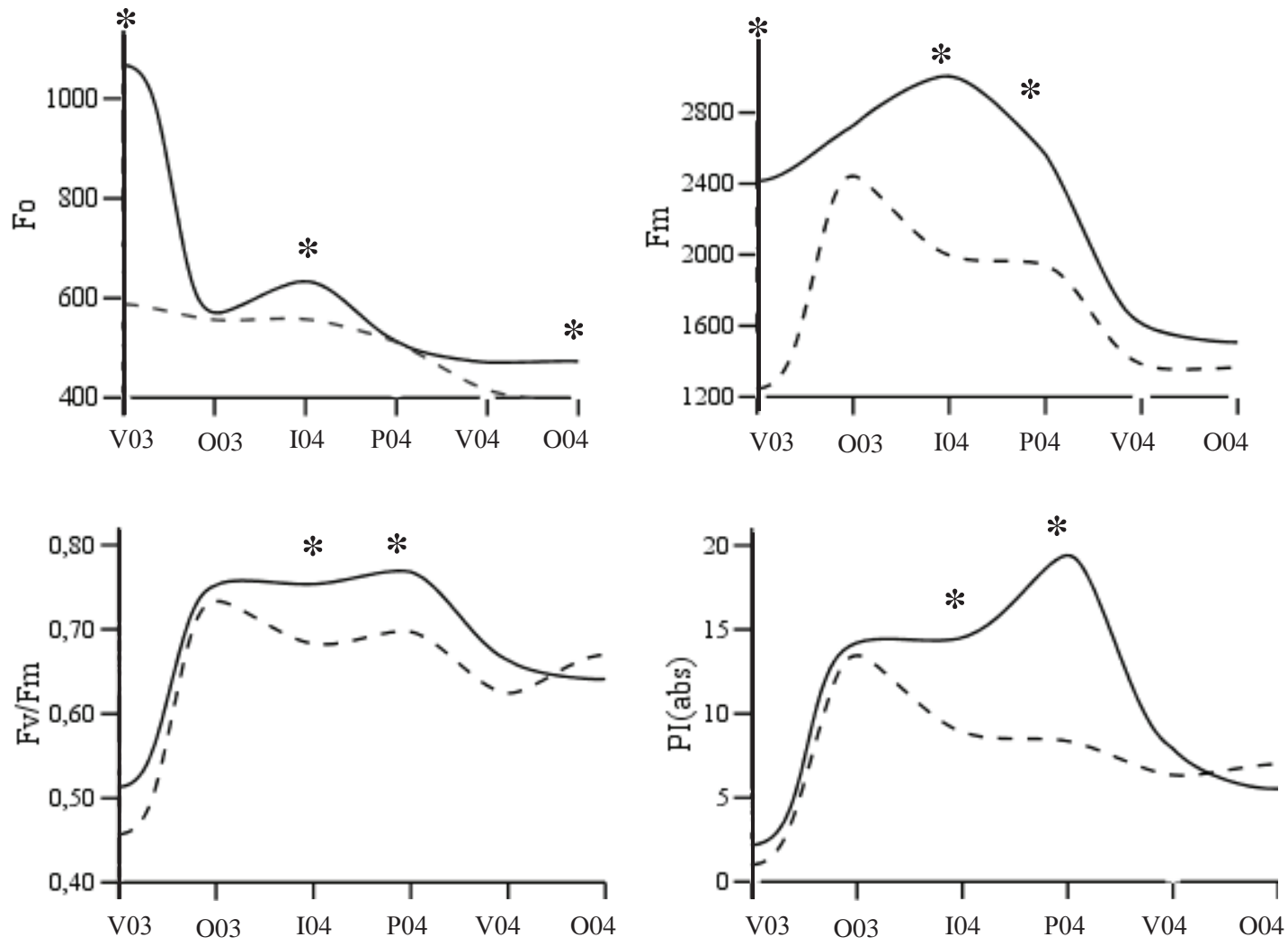

Figura 3. Evolución temporal del valor medio de los parámetros de fluorescencia de la clorofila $a$ para tratamientos agrupados en función de presencia/ausencia de tubo invernadero. Los controles señalados con “*” mostraron diferencias significativas $(P \leq 0,05)$. Controles. V03: verano 03; O03: otoño 03; I04: invierno 04; P04: primavera 04; V04: verano 04; O04: otoño 04. Curva discontinua: sin tubo; curva continua: con tubo.

Temporal evolution of chlorophyll $a$ fluorescence parameters mean values for treatments grouped according to presence / absence of tree shelters. Assessments dates marked “*” indicate significant differences at $P \leq 0.05$. Monitoring assessments: V03: summer 2003; O03: autumn 2003; I04: winter 2004; P04: spring 2004; V04: summer 2004; O04: autumn 2004. Dotted line: without tree shelter; solid line: tree shelter. 


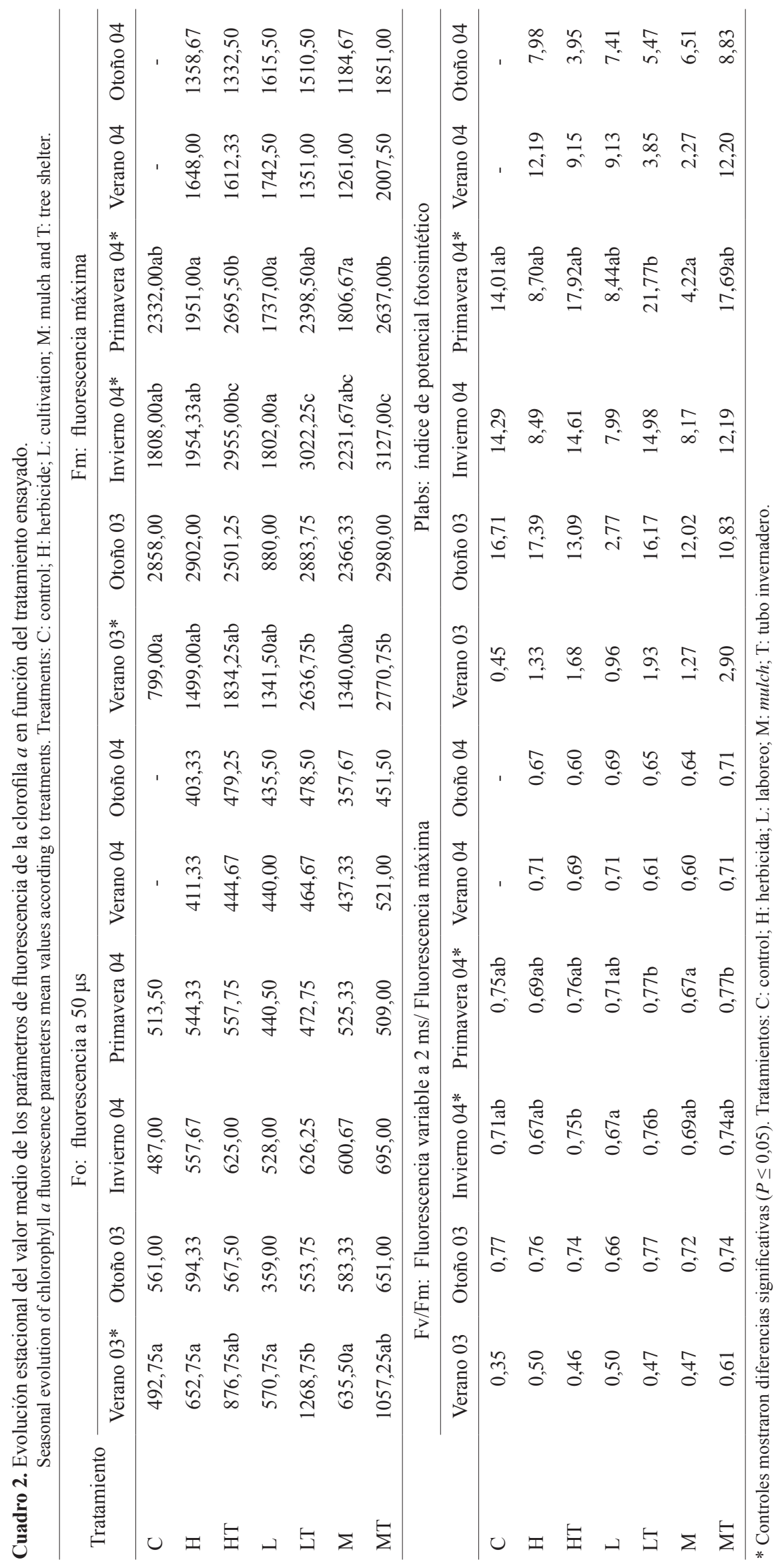


destacando la medida de verano. Los resultados de fluorescencia máxima $(\mathrm{Fm})$ mostraron los mayores valores asociados siempre al tratamiento con tubo invernadero, reflejando significación para las medidas de verano del 2003 y del invierno y la primavera del 2004.

La evolución temporal del índice de eficacia máxima fotoquímica $(\mathrm{Fv} / \mathrm{Fm})$ registró descensos acusados durante los periodos estivales, especialmente en el primer año de seguimiento (2003). Del mismo modo pudo observarse un ligero descenso asociado al periodo invernal, circunstancia que fue más palpable en los tratamientos sin tubo. En lo que respecta a las diferencias asociadas al factor tubo, se pudo comprobar su significación en los controles de invierno y de primavera, siendo los valores máximos los observados en los tratamientos combinados (figura 3).

El PIabs mostró un comportamiento similar al Fv/Fm, tanto en el cambio temporal como en la significación estadística asociada al factor tubo (periodos invernal y primaveral del año 2004). En este caso, se observaron claros descensos vinculados a los periodos estivales, así como un descenso en el control invernal para los tratamientos sin tubo. Se observó igualmente, un aumento del valor del índice en los tratamientos combinados durante la primavera del segundo año de seguimiento (figura 3).

Análisis de vitalidad de los brinzales. De acuerdo a la figura 4 se obtuvieron dos grupos de tratamientos claramente diferenciados, uno por encima y otro por debajo del valor medio de referencia. El grupo $+\Delta$ PI (abs) estuvo formado por los tratamientos combinados con tubo (LT, HT, MT) y el tratamiento individualizado con herbicida $(\mathrm{H})$. Este último, aunque obtuvo el valor mínimo, se incluyó junto con los tratamientos con tubo al registrar un incremento positivo del PIabs. Destacó especialmente el tratamiento MT que alcanzó un incremento superior al $50 \%$ (alto rendimiento). Por el contrario, el grupo con incrementos negativos incluyó a los tratamientos individualizados (C, M, L), destacando el tratamiento control, seguido del tratamiento $\mathrm{M}$.

Por otro lado, la correlación entre la fuerza motriz fotosintética $\Delta(\mathrm{DF}) \mathrm{rel}=\log$ (PIabs)rel como función del rendimiento relativo del transporte de electrones ((ETo/ ABS)rel) mostró que los datos se dispusieron siguiendo una línea recta (figura 5). Los datos mostraron que las plantas no protegidas presentaron los valores más bajos (cuadrante inferior izquierda) para la relación Log (PIabs) rel y (ETo/ABS)rel, mientras que las plantas protegidas mostraron los mayores valores (cuadrante superior derecha) para esa relación. Al realizar el análisis agrupado en función del factor tratamiento, los extremos segregaron los tratamientos en función de su vitalidad, destacando los resultados de MT (valores altos) y C (valores bajos) (figura 5). Estos resultados estaban en total concordancia con los derivados del análisis de desviación relativa del PIabs por tratamiento respecto del valor total de la parcela de ensayo (figura 4).

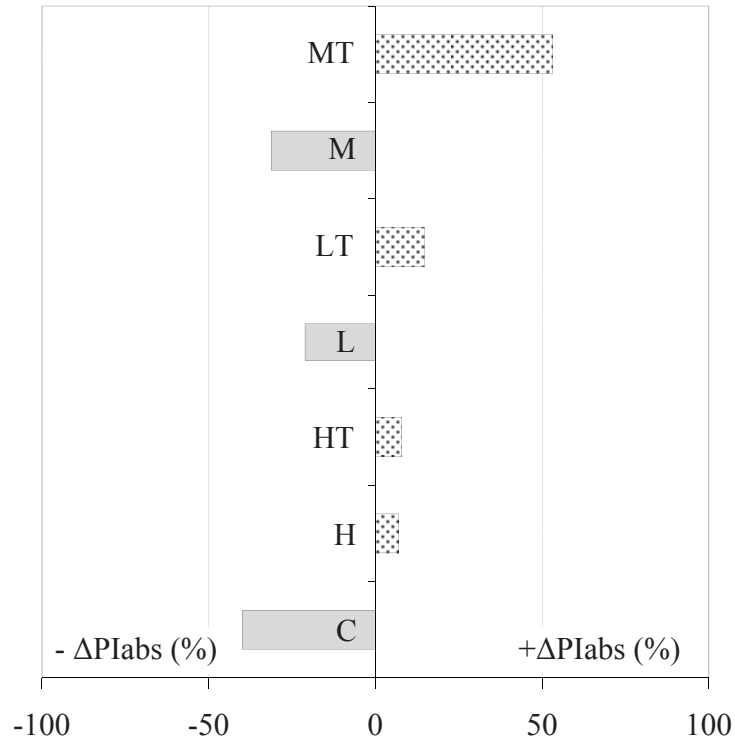

Figura 4. Desviación relativa del índice de potencial fotosintético (PIabs) en cada tratamiento respecto de la media total de medidas realizadas en la parcela experimental durante los dos años de seguimiento. Columnas grises: tratamientos con valor inferior al valor medio de parcela (- $\Delta$ PIabs (\%)). Columnas punteadas: tratamientos con valor superior al valor medio de parcela $(+\Delta$ PIabs $(\%))$. Abreviatura de los tratamientos en el cuadro 2.

Relative deviation of the performance index (PIabs) of each treatment from the average PIabs of all data in the experimental plot during the two-year monitoring. White bars: treatments with values bellow the average (- $\triangle \mathrm{PIabs}(\%))$. Dotted bars: treatments with values above the average $(+\Delta$ PIabs $(\%))$. Abbreviation of treatments in table 2.

\section{DISCUSIÓN}

Evolución estacional y evaluación de estrés mediante análisis de fluorescencia. De acuerdo con lo esperado, la evolución estacional de los parámetros de fluorescencia ha mostrado su utilidad para evaluar la respuesta temprana de brinzales de encina bajo diferentes condiciones de estrés ambiental. Se observó un descenso de la razón Fv/Fm en verano y en invierno, como consecuencia de las condiciones de estrés imperantes en ambos periodos (altas temperaturas, radiación y estrés hídrico en verano y fundamentalmente bajas temperaturas en invierno), y se obtuvieron aumentos de los valores del parámetro Fv/Fm en primavera y otoño, cuando dicho estrés era más reducido. Estos resultados coinciden con los obtenidos por diversos autores para estudios de encina en condiciones mediterráneas (Gratani et al. 2000, Ogaya y Peñuelas 2003, Oliveira y Peñuelas 2004, Baquedano y Castillo 2006). El valor medio del parámetro $\mathrm{Fv} / \mathrm{Fm}$ para las especies $\mathrm{C} 3$ en condiciones óptimas está en torno a 0,83 (Long et al. 1993). En este caso los resultados se mantuvieron en todo momento por debajo del umbral considerado como óptimo, lo que pondría de manifiesto cierto grado de estrés a lo largo de todo 


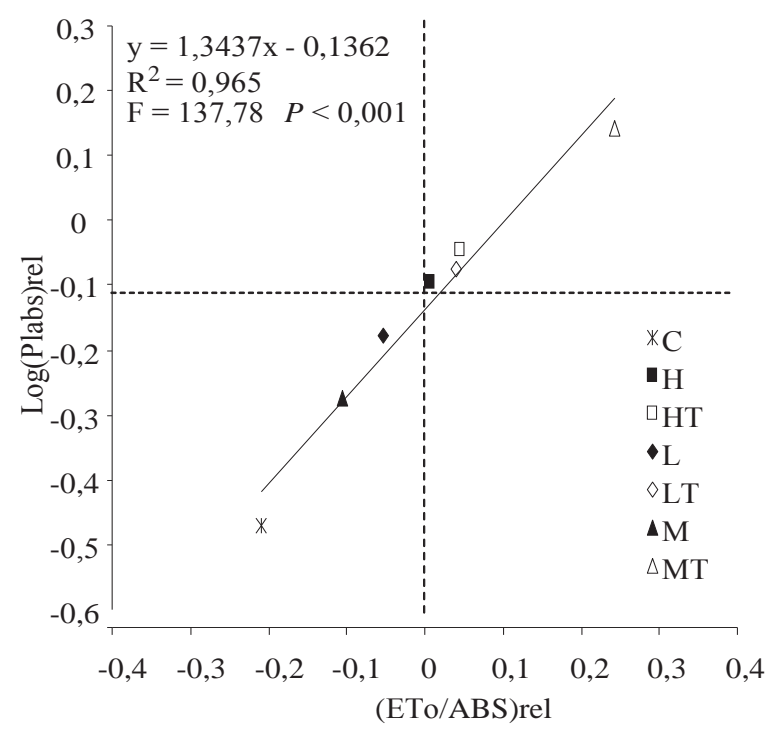

Figura 5. Correlación de la fuerza motriz fotosintética $\Delta$ (DFabs) rel $=\log$ (PIabs)rel como función de la eficiencia relativa del transporte de electrones fotosintético (ETo/ABS)rel para los diferentes tratamientos analizados durante los dos años de seguimiento. Líneas de referencia discontinuas representan los valores medios de cada parámetro para el conjunto de datos analizados. Abreviatura de los tratamientos en el cuadro 2.

Correlation of photosynthetic driving force $\Delta(\mathrm{DFabs}) \mathrm{rel}=\mathrm{Log}$ (PIabs)rel as a function of the relative yield of electron transport (ETo/ ABS)rel for different tested treatments. Dotted lines represent parameters mean values for all monitoring data. Abbreviation of treatments in table 2 .

el periodo de arraigo, siendo especialmente bajos durante el primer año de establecimiento ( $\mathrm{Fv} / \mathrm{Fm}=0,35-0,61)$.

Con carácter general, cualquier proceso de estrés puede producir una disminución de la relación $\mathrm{Fv} / \mathrm{Fm}$. Sin embargo, existen evidencias de la relativa insensibilidad al cambio que presenta este parámetro cuando se utiliza como el único factor para evaluar estrés en plantas (Strasser et al. 2000, Force et al. 2003). En este sentido, se define la fotoinhibición como un fenómeno dependiente de la luz (e influido por otros tipos de estrés) en los procesos fotoquímicos primarios, que es lentamente reversible y que suele ir acompañado por un aumento de la fluorescencia basal (Fo) (aunque no siempre es observado) y asociado con una inactivación en la población de centros funcionales de reacción del fotosistema II. A la fotoinhibición crónica, que generaría un daño permanente en el fotosistema II se la suele denominar fotoinactivación. Por otra parte, una caída (dependiente de la luz y en general de carácter rápidamente reversible) en la eficiencia en los procesos fotoquímicos, indicada por una disminución en la relación Fv/Fm, acompañado de un mantenimiento o caída de la señal de fluorescencia basal (Fo), indica un mecanismo de regulación o protector (fotoprotección) (Osmond et al. 1999, Tambussi 2005). En este caso, también aumenta la tasa de disipación térmica del exceso de energía a nivel del fotosistema II.
En este trabajo se observa una caída en la máxima eficiencia cuántica del fotosistema II ( $\mathrm{Fv} / \mathrm{Fm})$ durante el verano, acompañada por un aumento del valor de fluorescencia a $50 \mu$ s (Fo) y una reducción en la eficiencia del transporte de electrones (ETo/ABS). Este comportamiento es más evidente durante el verano del primer año de establecimiento, cuando las condiciones de estrés fueron mayores $\left(\mathrm{Fv} / \mathrm{Fm}_{\mathrm{v} 03}=0,35-0,61 ; \mathrm{Fo}_{\mathrm{v} 03}=492,75-1268,75\right.$; $\left.\mathrm{ETo} / \mathrm{ABS}_{\mathrm{V} 03}=0,12-0,27\right)$. El proceso de regulación a la baja (downregulation) del transporte de electrones en condiciones de estrés hídrico es un mecanismo de protección del aparato fotosintético bien conocido (Flexas et al. 2002). El incremento de la señal de fluorescencia basal Fo durante el estiaje (año 2003) podría apoyar la aparición de procesos de fotoinactivación. En condiciones de estrés hídrico serían necesarias bajas intensidades de radiación para saturar los sistemas fotosintéticos incrementando la susceptibilidad a la fotoinhibición (Lawlor 2002). En nuestro caso estos procesos serían inicialmente de carácter plástico, como muestra la recuperación observada para los diferentes parámetros de fluorescencia una vez desaparecido el estrés estival (medida de otoño). Las plantas sometidas a estrés, especialmente a estrés hídrico, aumentan su protección asociada a la disipación de energía por vía no fotoquímica incrementando la disipación de energía mediante calor, la mayoría del cual estaría relacionada con el ciclo de las xantofilas (Adams y Demming-Adams 1999, Manrique 2003). Sin embargo, algunos autores han destacado también para encina y coscoja (Quercus coccifera L.), la importancia de otros mecanismos protectores como la reacción de Mehler y la fotorrespiración (Baquedano y Castillo 2006).

Durante el invierno, los fenómenos de fotoinhibición y protección fueron, en términos generales, menos evidentes y aparecieron vinculados con mayor claridad a los tratamientos de control de la competencia estudiados. Conviene, sin embargo, indicar que discriminar entre diferentes tipos de estrés (hídrico, térmico, lumínico) puede resultar muy complicado. Aunque algunos autores (Osmond y Grace 1995) afirman que existen fenómenos que se presentan con mayor frecuencia vinculados a determinados tipos, como es el caso de la fotoinactivación del fotosistema II (asociada a una caída del valor de Fv/Fm) en plantas sometidas a frío.

Por otro lado, en concordancia con estudios precedentes en condiciones mediterráneas, durante el periodo primaveral se registraron los valores más altos de Fv/Fm, cuando la disponibilidad hídrica fue mayor (Valladares y Pearcy 2002, Ogaya y Peñuelas 2003). Algo similar ocurrió en la medida de otoño registrada el primer año.

Evaluación de los tratamientos de restauración mediante análisis de fluorescencia. Los tratamientos de control de la competencia no mostraron diferencias significativas claras entre los parámetros de fluorescencia de la clorofila $a$. Durante el invierno y la primavera, ésta última de manera especial, se obtuvo el mayor número de variables signifi- 
cativas desde el punto de vista de la diferenciación entre tratamientos, sin que se pudiera establecer una respuesta clara desde el punto de vista fotoquímico como respuesta a los diferentes tratamientos ensayados.

Sin embargo, el efecto del factor tubo invernadero sí se mostró decisivo en la interpretación de los resultados de fluorescencia de la clorofila $a$. Las plantas protegidas con tubo mostraron los mayores valores para todos los parámetros de fluorescencia analizados. Las diferencias entre las plantas protegidas y las no protegidas resultaron especialmente significativas durante el invierno y la primavera. Ogaya y Peñuelas (2003) observaron para encina que las hojas expuestas al sol tenían valores menores de la relación $\mathrm{Fv} / \mathrm{Fm}$ que las hojas de sombra. Por otro lado, Valladares et al. (2000) registran para esta misma especie, que el contenido de carotenoides y la disipación no fotoquímica del exceso de energía se incrementaba con el incremento de la radiación. Otros trabajos con plantas esclerófilas (Valladares y Pearcy 1997) muestran una fuerte fotoinhibición en hojas manipuladas para que su superficie estuviera expuesta a altas radiaciones y temperaturas. Estas diferencias entre sol y sombra podrían asimilarse al control que sobre la radiación ejerce el tubo invernadero, tal y como sugieren los resultados obtenidos en este trabajo. Según Bergez y Dupraz (2009) la transmitancia a la radiación fotosintéticamente activa de los tubos invernadero oscila entre el $35 \%$ y el $60 \%$, dependiendo del ángulo de incidencia de los rayos de luz. Por tanto, los resultados obtenidos pondrían de manifiesto que el efecto de protección del tubo invernadero limitaría la aparición de procesos de fotoinhibición/fotoprotección en las plantas protegidas, especialmente durante los periodos de invierno y primavera.

Durante el invierno se observó una caída significativa de la máxima eficiencia fotoquímica $(\mathrm{Fv} / \mathrm{Fm})$ en las plantas no protegidas, estando éste descenso asociado a una caída de fluorescencia máxima (Oliveira y Peñuelas 2004). Dicho comportamiento se suele vincular a procesos de fotoinhibición como mecanismo de protección de la planta frente al estrés invernal. Sin embargo, se pudo comprobar que la respuesta observada fue mantenida en el tiempo y no se registraron recuperaciones de los parámetros de fluorescencia hasta el otoño de 2004. La combinación de bajas temperaturas y altas irradiancias produce la llamada fotoinhibición crónica (fotoinactivación) que podría indicar un daño en el aparato fotosintético, y justificar los resultados observado en este trabajo. Aunque los fenómenos de fotoinactivación a menudo van acompañados de un incremento de Fo, no siempre se ha detectado este comportamiento. Por tanto, el tubo invernadero ejercería una triple acción de protección de los brinzales frente al estrés de invierno. En primer lugar, un control del exceso de irradiancia (Oliet y Jacobs 2007); en segundo, un control térmico que limitaría la desecación por acción del viento frío (Newton y McBeath 1996), y finalmente, incrementaría la temperatura en el entorno inmediato de la planta. Es un hecho conocido que la temperatura en el interior del tubo es superior a la del ambiente exterior (Navarro-Cerrillo et al. 2005, Bergez y Dupraz 2009). Según Gratani et al. (2000) la temperatura, tanto por exceso como por defecto, es otro factor determinante de la actividad fotoquímica de las plantas. De acuerdo con este autor, la tasa de transporte de electrones se reduce de repente por encima de $40^{\circ} \mathrm{C}$ y puede reducirse a la mitad de su valor máximo para temperaturas entre 8 y $10{ }^{\circ} \mathrm{C}$. En nuestro caso, las temperaturas medias ambientales para los meses invernales tuvieron un amplio rango de oscilación que varió entre 3 y $12{ }^{\circ} \mathrm{C}$ aproximadamente, mientras que las diferencias entre el interior y el exterior del tubo invernadero podrían alcanzar los $2^{\circ} \mathrm{C}$.

Durante la primavera, la protección del tubo frente a la radiación se convertiría en un elemento clave para la explicación de la respuesta observada, dada la menor incidencia de otros tipos de estrés (térmico e hídrico) durante ese periodo. Las plantas expuestas mostraban una caída más acusada de la relación $\mathrm{Fv} / \mathrm{Fm}$, que parecía asociada a un menor valor de fluorescencia máxima, lo que se ha vinculado con una interrupción del transporte de electrones en los centros de reacción (Baquedano y Castillo 2006). Sin embargo, como se ha puesto de manifiesto con anterioridad, los resultados de primavera podrían estar determinados por los procesos de fotoinhibición crónica detectados durante el invierno.

La mejora en el rendimiento del fotosistema II puede deberse a diversos fenómenos como el incremento en el número de centros de reacción funcionales, o porque ese fotosistema atrape la energía más eficientemente y presente un incremento en la eficiencia del transporte de electrones y una menor disipación de energía en forma de calor o fluorescencia (Ripley et al. 2004). Por tanto, en correspondencia con Force et al. (2003), el uso de diferentes parámetros de fluorescencia derivados del OJIP-test supone una ventaja para la evaluación del funcionamiento del fotosistema II frente al empleo exclusivo de parámetros individuales como Fv/Fm. Conjuntamente a lo anterior, y de acuerdo con Strasser et al. (2000), la vitalidad de una planta puede ser caracterizada de manera individual por el denominado índice de potencial fotosintético (PIabs). El PIabs refleja la funcionalidad de los fotosistemas I y II y proporciona información cuantitativa del estado de la planta bajo condiciones de estrés lumínico, hídrico, o térmico (Strasser et al. 2004, Strauss et al. 2006). De los parámetros derivados de OJIP-test, PIabs es considerado uno de los parámetros más sensibles a las condiciones de estrés, y muestra una mejor respuesta que $\mathrm{Fv} / \mathrm{Fm}$ cuando se considera individualmente (Gonçalves et al. 2007). Los resultados de este trabajo apoyan esta hipótesis (figura 3), donde las discrepancias entre plantas protegidas y no protegidas aparecen más acentuadas en el caso de PIabs que de Fv/Fm.

Finalmente, el análisis de vitalidad de la planta (figuras 4 y 5) ratifica los resultados observados a partir de los parámetros OJIP, lo que confirma la utilidad del índice de potencial fotosintético como factor de análisis de la vitalidad y de la calidad de la planta desde el punto de vista fotoquímico (Hermans et al. 2003, Gonçalves et al. 2007) y su 
aplicación a la evaluación temprana de técnicas dirigidas a mejorar el establecimiento en repoblaciones forestales. Las desviaciones de los valores de PIabs de cada individuo respecto del valor medio de referencia mostraron incrementos positivos $[+\Delta$ PIabs $]$ asociados principalmente al factor tubo invernadero (figura 4), llegando en algunos casos, como en el tratamiento MT a superar el $50 \%$, lo que ha sido considerado por determinados autores como individuos de alta vitalidad (Hermans et al. 2003). Por otra parte, la correlación entre Log (PIabs)rel y (ETo/ABS)rel, y, en concreto, la pendiente de la recta resultante, se considera como una propiedad específica del conjunto de plantas analizadas en relación con la transformación de la energía lumínica absorbida en energía química destinada a las reacciones metabólicas (Hermans et al. 2003). De acuerdo con los resultados obtenidos, las plantas protegidas mostraron mayor rendimiento fotoquímico que las no protegidas. Especialmente destacable fue el resultado para el tratamiento MT en correspondencia con lo observado en el análisis de desviación de los valores medios de $\mathrm{PI}(\mathrm{abs})$ por tratamiento.

\section{CONCLUSIONES}

El análisis de fluorescencia de la clorofila $a$ mediante los parámetros derivados del OJIP-test muestra ser eficaz para la evaluación temprana de la respuesta al estrés de establecimiento de brinzales de encina en condiciones mediterráneas. Los parámetros Fv/Fm y PIabs utilizados de forma conjunta muestran también su validez para la evaluación temprana de la respuesta fotoquímica de los brinzales ante diferentes técnicas de control de la competencia, aunque el PIabs presentó mayor sensibilidad para dicha evaluación que la relación Fv/Fm.

Los resultados de evaluación de las técnicas analizadas indican que los tratamientos de control de la competencia no produjeron cambios importantes de la fluorescencia de la clorofila. Sin embargo, la protección ejercida por el tubo invernadero sobre los brinzales limitó la aparición de procesos de fotoinhibición durante los periodos de primavera e invierno. La combinación de técnicas de control de la competencia y protección de los brinzales, especialmente, bajo el tratamiento mulch + tubo invernadero, muestran un mayor rendimiento fotoquímico que los tratamientos no combinados, carentes de protección. Por tanto, el análisis de fluorescencia de la clorofila $a$ es de gran interés y se muestra como una herramienta útil para la evaluación temprana de los efectos de determinados trabajos de restauración forestal, como la aplicación de técnicas que combinan el control de la competencia y la protección individualizada de los brinzales para mejorar el éxito de establecimiento de estos brinzales.

\section{AGRADECIMIENTOS}

Los autores agradecen al Centro de IFAPA "Alameda del Obispo", y en particular a su director Víctor Ortiz, las facilidades ofrecidas para el desarrollo de este estudio. Este trabajo de investigación se realizó con el apoyo de los proyectos INTERBOS (CGL2008-04503-C03-01/BOS) y AGL2009-12243-C02-02 "Variability, cataloguing, responses to stresses, and clonal propagation of Holm oak (Quercus ilex, subs. ballota) (DECOVA)" financiados por el Ministerio de Ciencia y Tecnología.

\section{REFERENCIAS}

Adams III W, B Demming-Adams, B Logan, D Barker, C Osmond. 1999. Rapid changes in xanthophyll cycle-dependent energy dissipation and photosystem II efficiency in two vines, Stephania japonica and Smilax australis, growing in the understory of an open Eucalyptus forest. Plant, Cell and Environment 22: 125-136.

Baquedano F, F Castillo. 2006. Comparative ecophysiological effects of drought on seedlings of the Mediterranean watersaver Pinus halepensis and water-spenders Quercus coccifera and Quercus ilex. Trees - Structure and Function 20: 689-700.

Bergez J, Z Dupraz. 2009. Radiation and thermal microclimate in tree shelter. Agricultural and Forest Meteorology 149: 179-186.

Christen D, S Schönmann, M Jermini, RJ Strasser, G Dèfago. 2007. Characterization and early detection of grapevine (Vitis vinifera) stress responses to esca disease by in situ chlorophyll fluorescence and comparison with drought stress. Environmental and Experimental Botany 60: 504-514.

Demmig-Adams B, W Adams III. 1992. Photoprotection and other responses of plants to high light stress. Annual Review of Plant Physiology and Plant Molecular Biology 43: 599-626.

Flexas J, J Bota, J Escalona, B Sampol, H Medrano. 2002. Effects of drought on photosynthesis in grapevines under field conditions: an evaluation of stomatal and mesophyll limitations. Functional Plant Biology 29: 461-471.

Force L, C Critchley, J van Rensen. 2003. New fluorescence parameters for monitoring photosynthesis in plants. Photosynthesis Research 78: 17-33.

Gonçalves J, J Santos, A Nina, L Chevreuil. 2007. Energetic flux and performance index in copaiba (Copaifera multijuga Hayne) and mahogany (Swietenia macrophylla King) seedlings grown under two irradiance environments. Brazilian Journal of Plant Physiology 19(3): 171-184.

Gratani L, P Pesoli, M Crescente, K Aichner, W Larcher. 2000. Photosynthesis as a temperature indicator in Quercus ilex L. Global and Planetary Change 24: 153-164.

Hermans C, M Smeyers, R Rodriguez, M Eyletters, R Strasser, J Delhaye. 2003. Quality assessment of urban trees: A comparative study of physiological characterization, airborne imaging and on site fluorescence monitoring by the O-J-IP-test. Journal of Plant Physiology 160: 81-90.

Lawlor D, G Cornic. 2002. Photosynthetic carbon assimilation and associated metabolism in relation to water deficits in higher plants. Plant, Cell and Environment 25: 275-294.

Long S, W Postl, H Bolhar-Nordenkampf. 1993. Quantum yields for uptake of carbon dioxide in C3 vascular plants of contrasting habitats. Planta 189: 226-234.

Maldonado-Rodríguez R. 1999. Biolyzer Software. Laboratory 
of Bioenergetics. University of Geneva, Switzerland.

Manrique E. 2003. Los pigmentos fotosintéticos, algo más que la captación de luz para la fotosíntesis. Ecosistemas 12(1): 49-59.

Martínez-Ferri E, L Balaguer, F Valladares, J Chico, E Manrique. 2000. Energy dissipation in drought-avoiding and droughttolerant tree species at midday during the Mediterranean summer. Tree Physiology 20: 131- 138.

Maxwell K, G Johnson. 2000. Chlorophyll fluorescente. A practical guide. Journal of Experimental Botany 51: 659-668.

Navarro Cerrillo RM, B Frageiro, C Ceacero, A del campo, R de Prado. 2005. Establishment of Quercus ilex L. subsp. ballota Desf. Samp. using different weed control strategies in Southern Spain. Ecological Engineering 25: 332-342.

Newton AC, C McBeath. 1996. The impact of desiccation on chlorophyll fluorescence in detached leaves of six tropical tree species. Photosynthetica 32: 491-501.

Niyogi KK. 1999. Photoprotection revisited: genetic and molecular approaches. Annual Review of Plant Physiology and Plant Molecular Biology 50: 333-359.

Ogaya R, J Peñuelas. 2003. Comparative field study of Quercus ilex and Phillyrea latifolia photosynthetic response to experimental drought conditions. Environmental and Experimental Botany 50: 137-148.

Oliet J, DF Jacobs. 2007. Microclimatic conditions and plant morph-physiological development within a tree shelter environment during establishment of Quercus ilex seedlings. Agricultural and Forest Meteorology 144: 58-72.

Oliveira G, J Peñuelas. 2004. Effects of winter cold stress on photosynthesis and photochemical efficiency of PSII of two Mediterranean Cistus albidus and Quercus ilex. Plant Ecology 175: 179-191.

Osmond C, J Anderson, M Ball, J Egerton. 1999. Compromising efficiency: the molecular ecology of light-resource utilization in plants. In Scholes JD ed. Physiological Plant Ecology. Press, Blackwell Science. p. 1-24.

Osmond CB, SC Grace. 1995. Perspectives on photoinhibition and photorespiration in the field: quintessential inefficiencies of the light and dark reactions of photosynthesis.
Journal of Experimental Botany 46: 1351-1362.

Ripley B, S Redfern, J Dames. 2004. Quantification of the photosynthetic performance of phosphorus-deficient Sorghum by means of chlorophyll-a fluorescence kinetics. South African Journal of Science 100 (11-12): 615-618.

Strasser R, A Srivastava, M Tsimilli-Michael. 2000. The fluorescence transient as a tool to characterize and screen photosynthetic samples. In Yunus M, U Pathre, P Mohanty eds. Probing Photosynthesis: Mechanisms, Regulation and Adaptation. Taylor and Francis, London. p. 445-483.

Strasser B, R Strasser. 1995. Measuring fast fluorescence transients to address environmental questions: The JIP-test. In Mathis P ed. Photosynthesis: from Light to Biosphere. Kluwer Academic Publishers, Dordrecht, The Netherlands. p. 977-980.

Strasser R, M Tsimilli-Michael, A Srivastava. 2004. Analysis of the fluorescence transient. In George C, C Papageorgiou, C Govindjee eds. Chlorophyll Fluorescence: A Signature of Photosynthesis. Advances in Photosynthesis and Respiration Series. Springer, Dordrecht. p. 321-362.

Strauss AJ, GHJ Kruger, RJ Strasser, PDR Van Heerden. 2006. Ranking of dark chilling tolerance in soybean genotypes probed by chlorophyll a fluorescence transient O-J-I-P. Environmental and Experimental Botany 56: 147-157.

Tambussi EA. 2005. Fotosíntesis, fotoprotección, productividad y estrés abiótico: algunos casos de estudio. Tesis doctoral. Barcelona. España. Universidad de Barcelona.

Valladares F, E Martínez-Ferri, L Balaguer, E Pérez-Corona, E Manrique. 2000. Low leaf-level response to light and nutrients in Mediterranean evergreen oaks: a conservative resource-use strategy?. New Phytologist 148: 79-91.

Valladares F, R Pearcy. 1997. Interactions between water stress, sun-shade acclimation, heat tolerance and photoinhibition in the sclerophyll Heteromeles arbutifolia. Plant, Cell and Environment 20: 25-36.

Valladares F, R Pearcy. 2002. Drought can be more critical in the shade than in the sun: a field study of carbon gain and photo-inhibition in a Californian shrub during a dry E1 Niño year. Plant, Cell and Environment 25: 749-759. 